\title{
STUDYING THE CONDUCTIVITY OF POLYSTYRENE USING 2-(SUB.)-5- [3-(5-NITRO-FUR-2-YL)-PROPEN-2-YLDIENE]AMINO -1,3,4- THIDIAZOLES AS ADDITIVES BY MEASURING FORBIDDEN ENERGY GAP
}

\author{
Emad Yousif", Mohammed Abdul Nabi ${ }^{* *}$, Ayad Hameed ${ }_{* * *}^{*}$ Nadia Salih ${ }^{*}$ and \\ Jumat Salimon*** \\ "Department of Chemistry, College of Science, Al-Nahrain University, Baghdad-Iraq. \\ ** Department of Physics, College of Science, Al-Nahrain University, Baghdad-Iraq. \\ School of Chemical Science and Food Technology, Faculty of Science and \\ Technology, University Kebangsaan Malaysia, 43600 Bangi, Selangor, Malayysia.
}

\begin{abstract}
The optical properties of Polystyrene in the region from $(200-900 \mu \mathrm{m})$ were studied using UV-VIS spectrophotometer by preparing composites with different organic materials. The optical data analyzed and interpreted in term of the theory of phonon assisted indirect electronic transition. It is observed that the energy gap of the samples, affected by the type of organic doping, and increase the conductivity of the polystyrene.
\end{abstract}

\section{Introduction}

There has been growing interest in the characterization of modified polymers. Great attention has been focused on enhancing their electrical conduction and improving their optical properties. The optical characteristics are extremely important not only for scientific knowledge but also for modern technology applications.

The plastics proved to be very useful, with a wide range of applications stemming from their toughness, plasticity, strength to weight ratio, low cost and ease of fabrication. They also had a very high electrical resistance. Indeed, one of the main uses for these materials was as insulators. Right up to the 1950 s, the very notion of electrically conducting polymers was considered laughable $^{(1)}$. In 1958 , polyacetylene was first synthesized by Natta et al. ${ }^{(2)}$ as a black powder. This was found to be a semiconductor with conductivity between $7 \times 10^{-11}$ to $7 \times 10^{-3} \mathrm{Sm}^{-1}$, depending upon how the polymer was processed and manipulated ${ }^{(3)}$.

The information about polystyrene structure has been mostly accumulated from the study of optical properties in a wide wavelength range. The significance of polystyrene is in its energy gap ${ }^{(4)}$. The best definition of the energy gap is the minimum energy difference between the lowest minimum of conduction band and the highest maximum of the valance band ${ }^{(5)}$. Two types of optical transitions are direct and indirect transitions, both involve the interaction of an electromagnetic wave with the electron in valance band with may cross the forbidden gap to the conduction band ${ }^{(6)}$.

Indirect transition possible only by phonon assisted transition. The value and shape of the mobility gap in polystyrene depend on the proportion conditions such as substrate temperature, degree of impurity and defects of the material. Any variation is such parameters lead to a shift in the absorption edge towards higher or lower energy ${ }^{(7)}$. The absorption coefficient is defined $\mathrm{be}^{(8)}$, which can be determined from the normal incidence transmission through plane-parallel plate of thickness (d) interference and multiple reflection are neglected. The reflectance (R) and transmittance $(\mathrm{T})$ are related by the equation (1)

$$
\mathrm{T}=\mathrm{B}(1-\mathrm{R})^{2} \exp (-\alpha \mathrm{d}) \text {. }
$$

Where $\mathrm{B}$ is a constant. This equation can be rewritten as in equation (2)

$$
\alpha \mathrm{d}=2.303 \mathrm{~A}+\operatorname{Ln}\left[\mathrm{B}(1-\mathrm{R})^{2}\right]
$$

$A$ is the optical absorbance, $(A=-\log T)$.

So the absorption coefficient $\alpha(\omega)$ in term of absorbance become:-

$$
\alpha(\omega)=2.303(\mathrm{~A} / \mathrm{d}) .
$$

For direct band-to-band transition, the energy dependence of absorption coefficient is of the form:- 
$\alpha=\alpha_{0}(\mathrm{~h} v-\mathrm{Eg})^{\mathrm{r}} / \mathrm{h} v$

Where (hv) is the photon energy and (Eg) is the energy gap, is constant depending on the type of the electronic transitions. It takes the value $(1 / 2)$ for allowed direct transition and (3/2) for forbidden direct transition and the absorption coefficient $\left(\alpha \geq 10^{4} \mathrm{~cm}^{-1}\right)$.

The energy dependence absorption coefficient is of the from:-

$$
\alpha=\alpha_{0}(\mathrm{~h} v-E g \pm E p)^{\mathrm{r}} / \mathrm{h} v
$$

Where (Eg) is the minimum energy gap, (Ep) is the phonon, $(+)$ absorbed (-) emitted, (r) take the value of It takes the value (2) for allowed indirect transition and (3) for forbidden indirect transition and the absorption coefficient $\left(\alpha<10^{4} \mathrm{~cm}^{-1}\right)^{(9)}$

By plotting $(\alpha h v)^{1 / r}$ versus (hv) for fixed (r) value, the extrapolation of the liner part could be used to define $\mathrm{Eg}^{(9)}$. On a viewpoint of excited electrons, the valence band maximum and conduction band minimum occur at the zone center $(\Delta \mathrm{k}=0)$, in direct band gap materials, where $(\mathrm{k}=2 \pi / \lambda)$. So upward transition of electrons does not require a change in momentum, or the involvement of a phonon. In the direct band gap materials, an electron raised to the conduction band, by photon absorption, will settle there for a very short time and the recombination again with a valance band hole resulting in emission of light with energy equal to the band gap. Thus, the probability of radiactive recombination is very high in direct band gap semiconductors ${ }^{(10)}$

On the contrary, in the indirect band gap semiconductors, the valence band maximum and conduction band minimum occur at different zone center $(\Delta k \neq 0)$. Upward or downward transition of electrons requires a change in momentum or involvement of a phonon. In consequence an unassisted recombination between an electron and hole is very unlikely. When an electron is mobile in a semiconductor, it may encounter a defect and become trapped or otherwise de-exited. This process of non-radiactive recombination quenches luminescence. Hence, indirect semiconductor tend to both slow in response (the carrier lifetime is long) and inefficient as light emitter. Thus, an electron dwelling in the conduction band minimum, at $(\Delta \mathrm{k} \neq 0)$, cannot recombine with a hole at $(\Delta \mathrm{k}=0)$ until a phonon with right energy and momentum is available $^{(10)}$.

Polystyrene in general are transparent and colorless. The existence of conductive additive raising the transition wavelength into visible region and the optioned lighter structure is very useful in some specific advanced technology, such as electrochromic displays ${ }^{(8)}$. Optical switching and rechargeable batteries $^{(11)}$.

\section{Experimental}

2-(sub.)-5-[3-(5-nitro-fur-2-yl)-propen-2-

yldiene]amino -1,3,4-thidiazoles [1-5]. were prepared by the method described by Hameed et al. ${ }^{(12) \text {. }}$

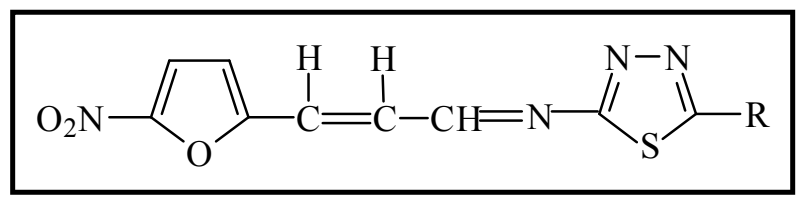

\section{Purification of Polystyrene ${ }^{(13)}$}

Commercial Polystyrene (PS) with average number molecular weight $\left(2 \times 10^{5}\right)$ determined by viscometric technique ${ }^{(14)}$ was freed from additives by re-precipitation twice from chloroform solution in ethanol. The purified polymer was dried under reduced pressure at room temperature for 24 hours.

\section{Films Preparation}

$0.5 \%$ concentrations of polystyrene solutions in chloroform was used to prepare 30 micrometer thickness of polymer films, (measured by a micrometer type 2610 A, Germany) with out and with $0.5 \%$ of the metal complexes prepared. The films were prepared by evaporation technique at room temperature for 24 hours. To remove the possible residual chloroform solvent, film samples were further dried at room temperature for three hours under reduced pressure. The optical absorbance (A) of the sample were measured as a function of wavelength $(\lambda)$ ranged from 200 to $900 \mathrm{~nm}$ by using Shimadzu UV-VIS 160A-Ultraviolet-spectrophotometer. The instrument is computerized with a full scale absorbance up to (2.5). The light sources are halogen lamp and socket-deuterium 
lamp. The detector is Si-photodiode and all measurements were performed at room temperature. UV/VIS absorption spectroscopy were made for prepared samples before and after conjunction. The spectra were used to carry out the energy gap by plotting $(\alpha h v)^{2}$ versus $(\mathrm{h} v)$ with $(\mathrm{r})$ value $(1 / 2)$. The linear portion was best fitted with $(\mathrm{r}=1 / 2)$, which indicates a transition of direct type. Energy gap shift for all samples was plotted as a function to conjunction type.

\section{Results and Discussion}

The relation between $(\alpha h v)^{2}$ versus photon energy for the Polystyrene samples are shown in Fig.(1 to 6) for allowed transition.

For doped samples shown in Figs. (2 to 6), the shift in the energy gap could be attributed to the formation of polorans in the doped films ${ }^{(15,16)}$. The evidence of Polaron formation is made that the reaction in band to band transition due to shifting the band density of state toward the energy gap (see Fig. (8)). This observation is not similar to the doping in conventional semiconductors when the band to band absorption strength are not affected by the formation of dopant state in the energy gap (17)

The effect of the organic addition on the values of phonon energies is also investigated and the result are shown in Table (1).
The results presented in Figs. (2 to 6) indicate the existence of two polaron bands in the energy gap. The first one represents the transition from valance band to bonding polaron band. The second band represents the transition from valance band to anti-bonding polaron band, which is in a good agreement with results obtained by Bredas et al. ${ }^{(18)}$ and with Pratt ${ }^{(19)}$. Conductivity measurement ${ }^{(20)}$ of PS with and without additive can obtained by adopting the data of energy gap, (see Table (1) and Figs. from (1 to 6)). The conductivity measurements for PS in the presence of additive increase in the following order.

B, 5, 4, 3, 2, 1

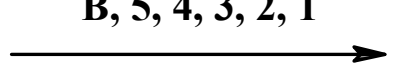

Table (1)

Indicate the energy band gap according to the direct allowed transition.

\begin{tabular}{|c||c|}
\hline Sample & Eg (eV) \\
\hline \hline $\mathrm{B}$ & 4.2 \\
\hline \hline 1 & 3.5 \\
\hline 2 & 3.7 \\
\hline \hline 3 & 3.8 \\
\hline \hline 4 & 3.9 \\
\hline 5 & 4 \\
\hline
\end{tabular}

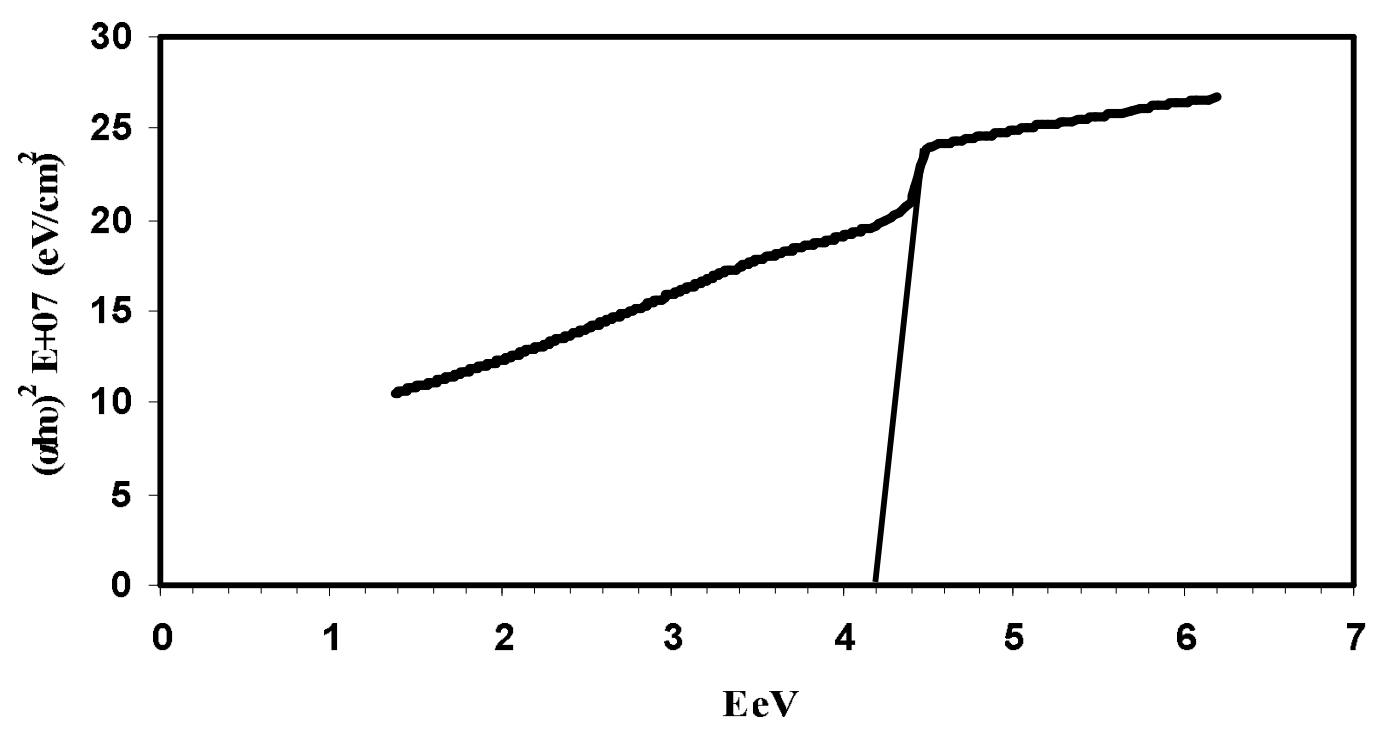

Fig.(1) : Allowed direct transition (ahw) ${ }^{2}$ Vs. Energy for the sample (B). 


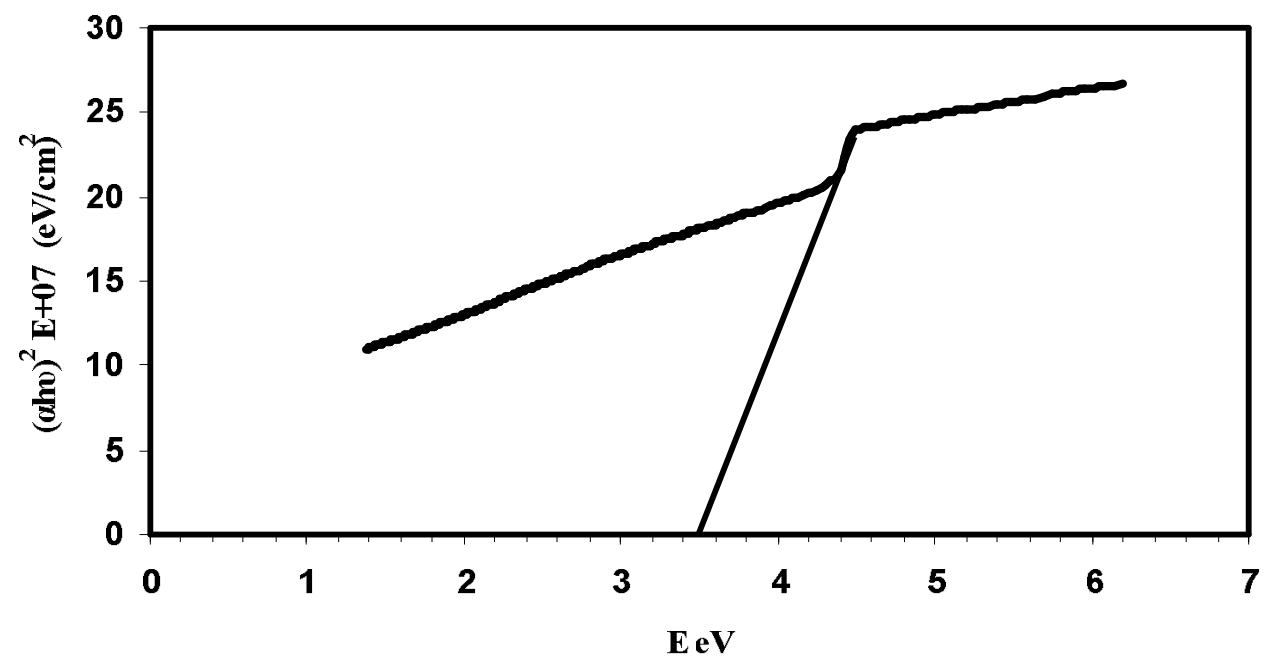

Fig.(2) : Allowed direct transition (ahw) ${ }^{2}$ V. Energy for the sample (1).

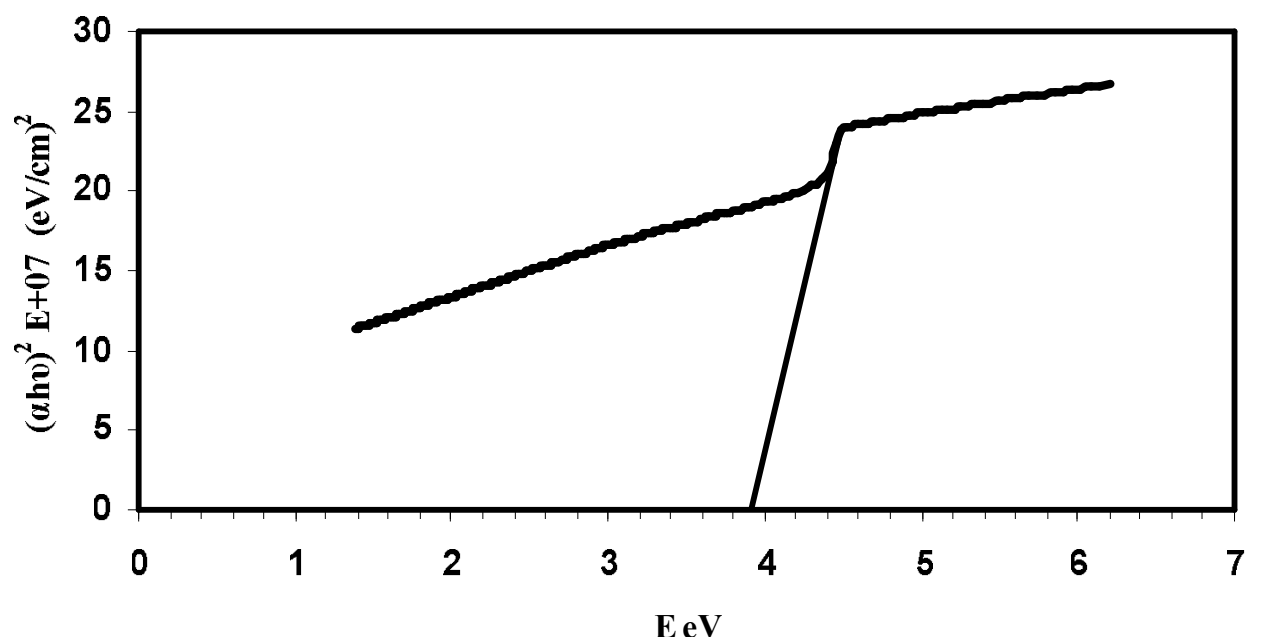

Fig.(3) : Allowed direct transition (ahw) ${ }^{2}$ Vs. Energy for the sample (2).

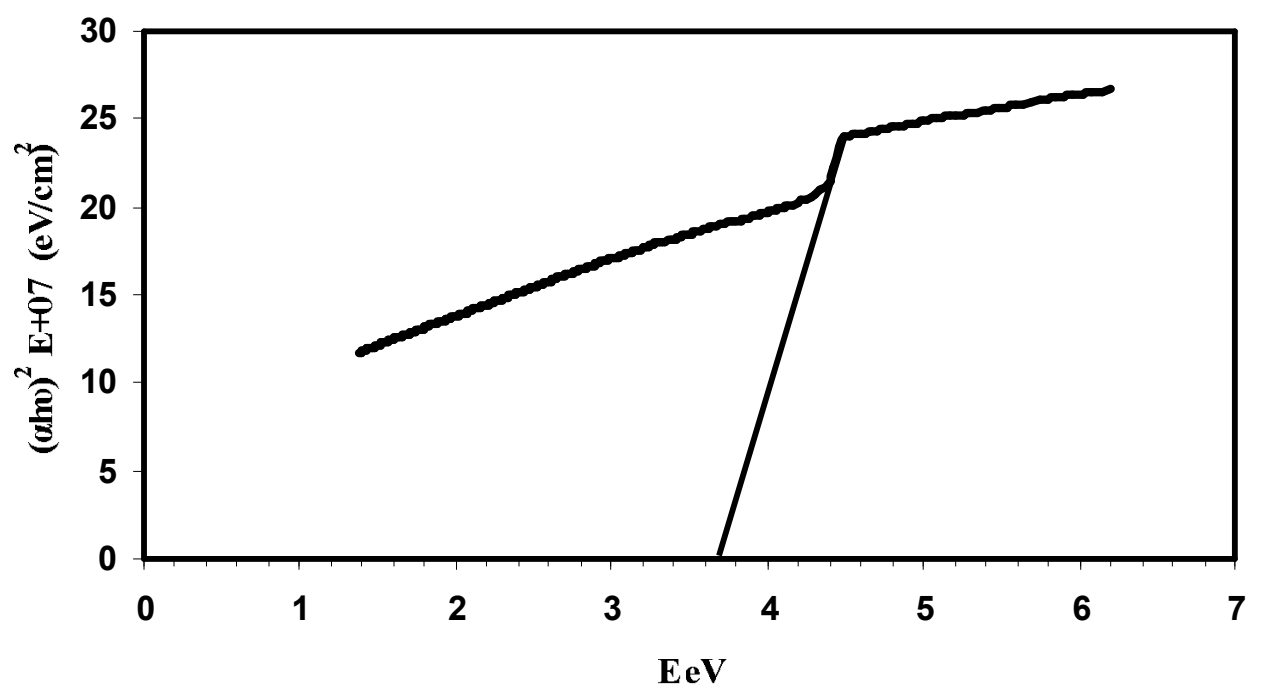

Fig. (4) : Allowed direct transition (ahw) ${ }^{2}$ Vs. Energy for the sample (3). 


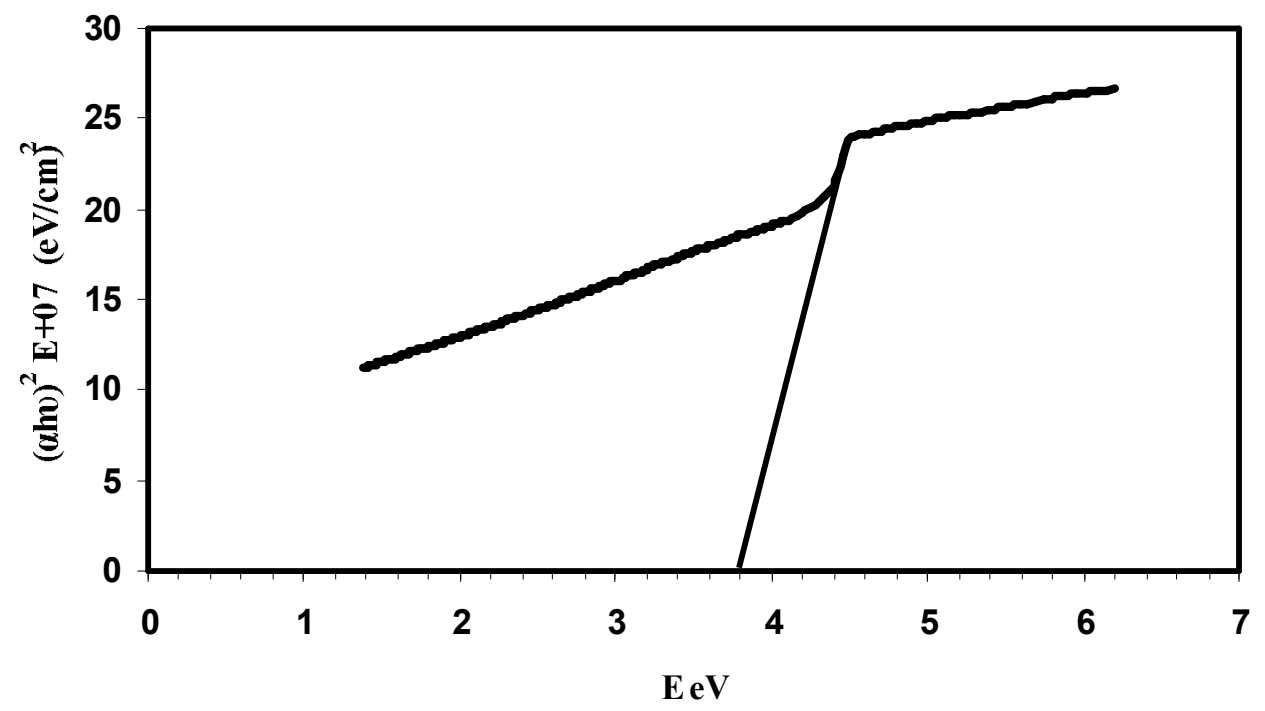

Fig.(5) : Allowed direct transition (ahw) ${ }^{2}$ Vs. Energy for the sample (4).

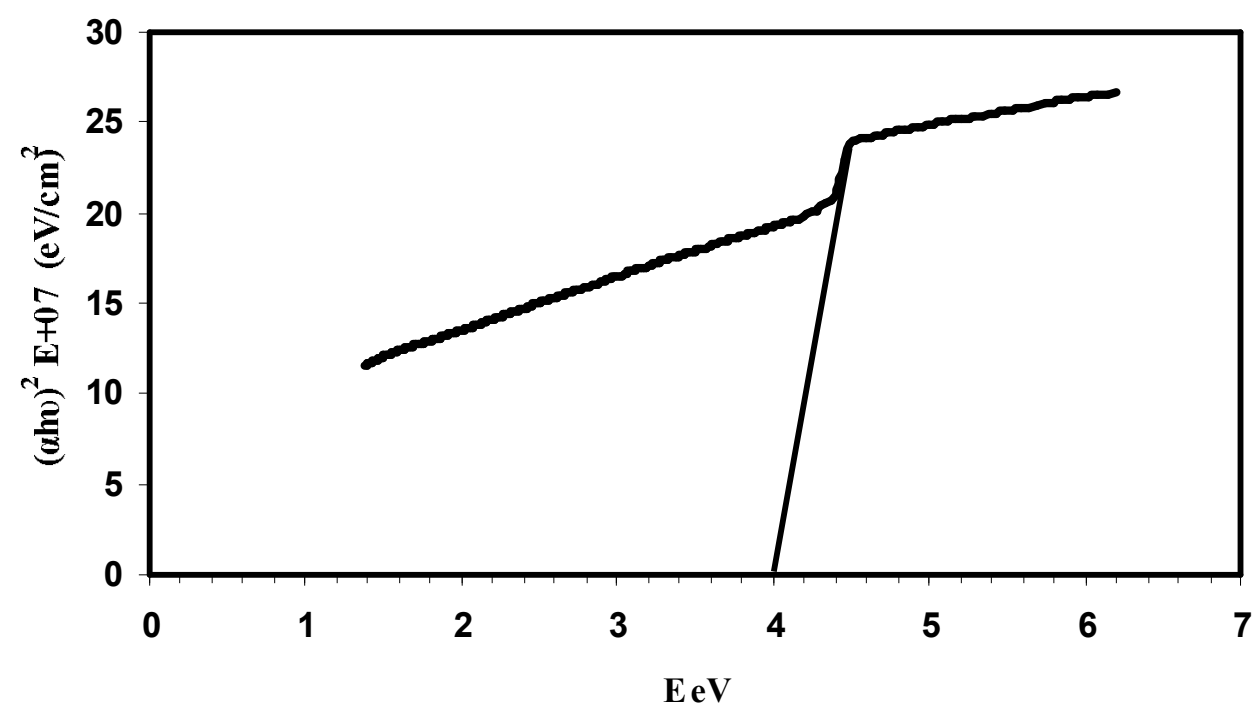

Fig.(6) : Allowed direct transition (ahw) ${ }^{2}$ V. Energy for the sample (5).

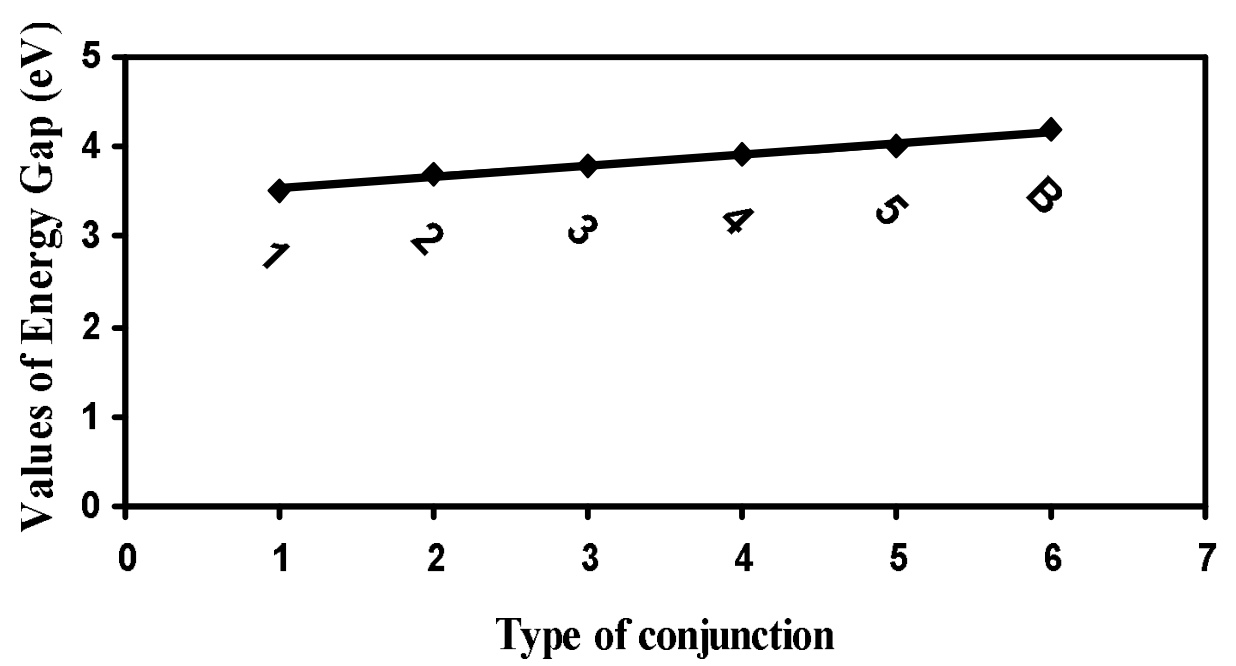

Fig.(7) : Indicate the Energy gap according to the type of conjunction. 


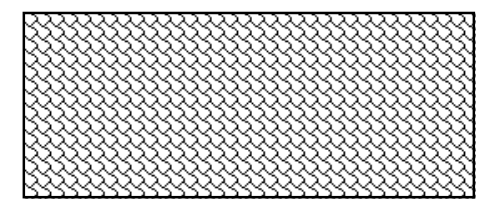

\section{Conduction}

Band
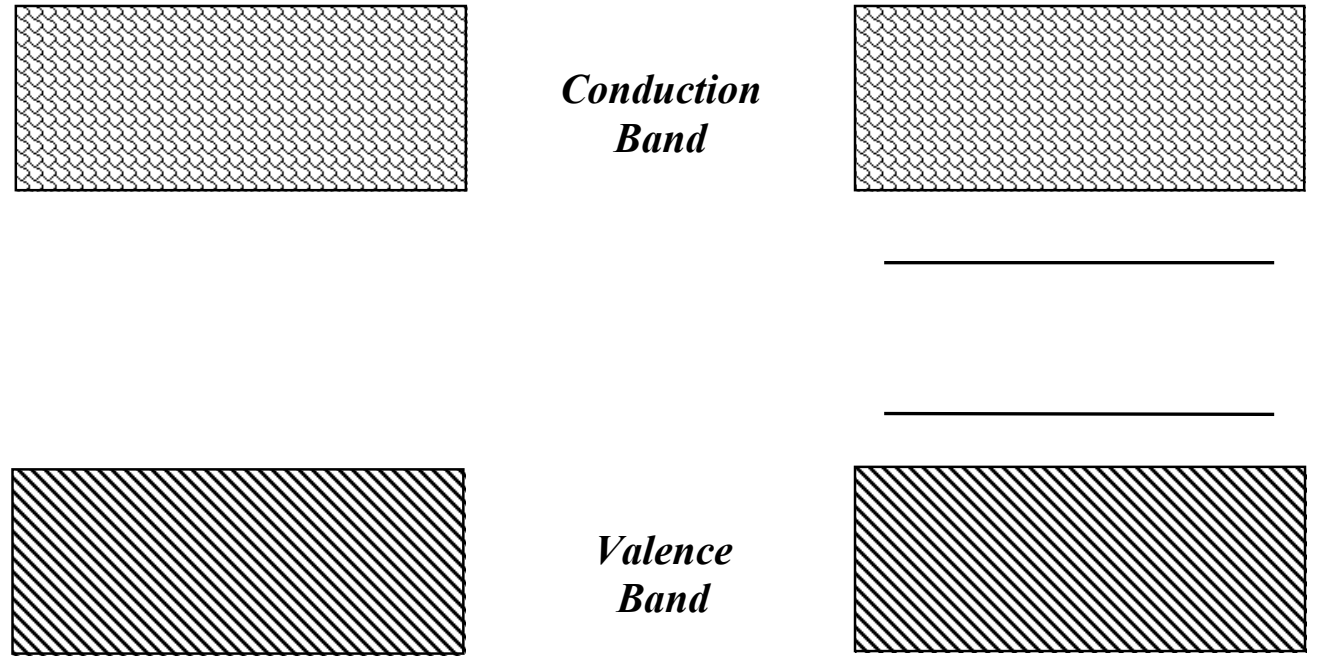

Valence

Band

Natural Polystyrene

Polaron

\section{Fig.(8) : Indicate the Conduction band and the Valence band for the pure and conjuncted polystyrene.}

\section{References}

[1] R. B. Kaner, A. G. MacDiarmid, Scientific America, 60(February) 1988.

[2] G. Natta, G. Mazzanti and P. Corradini, Atti. Acad. Naz. Lincei, Cl. Sci. Fis. Mat. Rend., 8(1958) 25.

[3] M. Hatano, S. Kambara, S. Okamoto, J. Polym. Sci., 26(1961) 51.

[4] K. Abass, H. L. Mansour and N. F. Habubi," J. Math. Phys.", 12(1991) 78.

[5] J. Tauc," Amorphous and Liquid Semiconductor", Plenum. Press, London, New York, (1974).

[6] N. F. Mott, E. A. Davis," Electrical Processes in non-Crystalline Materials", Clarendon Press, Oxford, (1971).

[7] F. A. Jenkins and H. E. White, "Fundmantals of Optics", $4^{\text {th }}$ edition, (1981).

[8] K. Kaneto, T. Shiraishi and Y. luishi," Jap. J. Appl. Phys.", 22(1983)412.

[9] S. Ayman, Ph.D. thesis., "University of Baghdad, (1997).

[10] www.inf.vtt.fi/pdf/chapter.1/2000.

[11]K. Kaneto, T. Shiraishi and K. Luishi, "Jap. J. Appl .phys",24 (1985)107.
[12]A. S. Hameed, E.A. Yousif and M. A. Sabbah "Journal of Al-Nahrain University", 7(2005)6.

[13]O. Taha, "Ph.D. thesis, Al Mustansyira University" (2002).

[14]R. Houwink, J prakt. Chem. 15 (1940)157.

[15]G. Frand, G. Roussel, O. Bohnke,"J. Electro Chem.Soc.", 43 (1992)1738.

[16]J. Daly and R. A. Pethrick, "Polymer", 22(1981)37.

[17]L. Ding, T. Sih, G. Yong and S. Dong, "Polym.J.", 29(1988)30.

[18]J. Bredas, J. C. Scott, K. Yakushi and C. B. Streat , "Phys.Rev.", 30B (1984)1023.

[19]C Pratt - homepage.ntlworld.com (2006). W. A. Abdul Chaffer and W. A. Hussain, "J. Basrah Researches", 25(2000)22. 